\title{
A prevalência de internações hospitalares por diarreia e gastroenterite de origem infecciosa em crianças de 0 a 4 anos no município de Macaé/RJ
}

The prevalence of hospital admissions for diarrhea and infectious gastroenteritis in children aged 0 to 4 years in the city of Macaé/RJ

Prevalencia de ingresos hospitalarios por diarrea y gastroenteritis infecciosa en niños de 0 a 4 años de la ciudad de Macaé/RJ

Kelly Pinheiro Vieira ORCID: https://orcid.org/0000-0002-5774-4612 Universidade Federal do Rio de Janeiro, Brasil E-mail: kellypinheirov@gmail.com

Patrícia Regina Affonso de Siqueira ORCID: https://orcid.org/0000-0002-8782-7048 Universidade Federal do Rio de Janeiro, Brasil E-mail: patriciapras@gmail.com

Rejane Correa Marques ORCID: https://orcid.org/0000-0001-6730-7769 Universidade Federal do Rio de Janeiro, Brasil E-mail: rejanecorreamarques@gmail.com Isis Vanessa Nazareth ORCID: https://orcid.org/0000-0002-2504-2472 Universidade Federal do Rio de Janeiro, Brasil E-mail: ivnenfermagem@gmail.com

Fabrícia Costa Quintanilha Borges ORCID: https://orcid.org/0000-0002-5871-4407 Secretaria Municipal de Saúde de Macaé, Brasil E-mail: fafaquinta05@gmail.com

Glaucimara Riguete de Souza Soares ORCID: https://orcid.org/0000-0002-2211-7854 Universidade Federal do Rio de Janeiro, Brasil E-mail: glau_riguete@hotmail.com

Sabrina Ayd Pereira Jose ORCID: https://orcid.org/0000-0003-1032-9259 Universidade Federal do Rio de Janeiro, Brasil E-mail: sabrinaayd@gmail.com

Isadora Oliveira do Amaral ORCID: https://orcid.org/0000-0003-1641-7188 Universidade Federal do Rio de Janeiro, Brasil E-mail: isadora.o.amaral@gmail.com

Pollyana Abreu Campos da Cruz ORCID: https://orcid.org/0000-0002-8059-3242 Universidade Federal do Rio de Janeiro, Brasil

E-mail: pollycamposdacruz@hotmail.com

\begin{abstract}
Resumo
Objetivos: Descrever e discutir o número de internações hospitalares por diarreias e gastroenterites de origem infecciosa em crianças de 0 a 4 anos em Macaé/RJ, comparado ao Estado do Rio de Janeiro e ao Brasil. Metodologia: Pesquisa epidemiológica com abordagem quantitativa com análise retrospectiva a partir de dados de fontes secundárias. A coleta de dados ocorreu no período compreendido entre julho de 2020 e maio de 2021 , analisando o período de 2016 a 2020. Resultados: No município de Macaé em 2016, 4 crianças foram internadas por alguma doença diarreica e gastroenterite de origem infecciosa; 6 crianças em 2017; 4 crianças em 2018; 3 crianças em 2019 e 2 crianças em 2020. No estado do Rio de Janeiro em 2016, 1.046 crianças foram internadas por alguma doença diarreica e gastroenterite de origem infecciosa; 959 crianças em 2017; 1.176 crianças em 2018; 1.362 crianças em 2019 e 1.427 crianças em 2020. No Brasil em 2016, 45.239 crianças foram internadas por alguma doença diarreica e gastroenterite de origem infecciosa; 34.940 crianças em 2017; 33.499 crianças em 2018; 31.777 crianças em 2019 e 18.234 crianças em 2020. Conclusão: Nas plataformas públicas de informações sobre morbimortalidade infantil,
\end{abstract}


Macaé apresentou grandes diferenças nos padrões de internação infantil se comparado com os dados estaduais e nacionais que apresentam números expressivos de crianças adoecendo por esta causa evitável. Assim, faz se necessário a ampliação de investigações no que se refere às internações infantis por diarreia e gastroenterite de origem infecciosa.

Palavras-chave: Saúde da criança; Saneamento básico; Meio ambiente; Saúde pública.

\begin{abstract}
Objective: To describe and discuss the number of hospital admissions for diarrhea and gastroenteritis of infectious origin in children aged 0 to 4 years in Macaé/RJ, compared to the State of Rio de Janeiro and Brazil. Methodology: Epidemiological research with a quantitative approach with retrospective analysis based on secondary data. Data collection took place between July 2020 and May 2021, analyzing the period from 2016 to 2020. Results: In the city of Macaé in 2016, 4 children were hospitalized for some diarrheal disease and gastroenteritis of infectious origin; 6 children in 2017; 4 children in 2018; 3 children in 2019 and 2 children in 2020. In the state of Rio de Janeiro in 2016, 1,046 children were hospitalized for some diarrheal disease and gastroenteritis of infectious origin; 959 children in $2017 ; 1,176$ children in 2018; 1,362 children in 2019 and 1,427 children in 2020. In Brazil, in 2016, 45,239 children were hospitalized for some diarrheal disease and gastroenteritis of infectious origin; 34,940 children in 2017; 33,499 children in 2018; 31,777 children in 2019 and 18,234 children in 2020. Conclusion: On public information platforms on child morbidity and mortality, Macaé showed large differences in child hospitalization patterns compared to state and national data that show significant numbers of children falling ill from this preventable cause. Thus, it is necessary to expand investigations with regard to childhood hospitalizations for diarrhea and gastroenteritis of infectious origin.
\end{abstract}

Keywords: Child health; Basic sanitation; Environment; Public health.

\title{
Resumen
}

Objetivo: Describir y discutir el número de ingresos hospitalarios por diarrea y gastroenteritis de origen infeccioso en niños de 0 a 4 años en Macaé / RJ, en comparación con el Estado de Rio de Janeiro y Brasil. Metodología: Investigación epidemiológica con enfoque cuantitativo con análisis retrospectivo basado en datos secundarios. La recolección de datos se realizó entre julio de 2020 y mayo de 2021, analizando el período de 2016 a 2020. Resultados: En la ciudad de Macaé en 2016, 4 niños fueron hospitalizados por alguna enfermedad diarreica y gastroenteritis de origen infeccioso; 6 niños en 2017; 4 niños en 2018; 3 niños en 2019 y 2 niños en 2020. En el estado de Río de Janeiro en 2016, 1.046 niños fueron hospitalizados por alguna enfermedad diarreica y gastroenteritis de origen infeccioso; 959 niños en 2017; 1.176 niños en 2018; 1.362 niños en 2019 y 1.427 niños en 2020. En Brasil, en 2016, 45.239 niños fueron hospitalizados por alguna enfermedad diarreica y gastroenteritis de origen infeccioso; 34,940 niños en 2017; 33,499 niños en 2018; 31,777 niños en 2019 y 18,234 niños en 2020. Conclusión: En las plataformas de información pública sobre morbilidad y mortalidad infantil, Macaé mostró grandes diferencias en los patrones de hospitalización infantil en comparación con los datos estatales y nacionales que muestran un número significativo de niños que enferman por esta causa prevenible. Por lo tanto, es necesario ampliar las investigaciones con respecto a las hospitalizaciones infantiles por diarrea y gastroenteritis de origen infeccioso.

Palabras clave: Salud del niño; Saneamiento básico; Medio ambiente; Salud pública.

\section{Introdução}

A diarreia é a segunda causa de morte em crianças menores de 5 anos no mundo inteiro. No último relatório da Organização Mundial da Saúde (OMS) com o Fundo das Nações Unidas para a Infância (UNICEF), com o Grupo Banco Mundial e com as Nações Unidas, estima-se que $8 \%$ das crianças no planeta morrem por doença diarreica. Ações vêm sendo desenvolvidas em todo o mundo e os números vêm diminuindo desde 2000, porém, mesmo com os avanços alcançados no combate a doenças infantis, as de origem infecciosas permanecem sendo a principal causa de morte entre crianças (UN IGME, 2019).

As doenças diarreicas agudas (DDA) são um grupo de doenças infecciosas que atingem o sistema gastrointestinal e são caracterizadas por um conjunto de sinais e sintomas, sendo eles: diminuição da consistência das fezes, número elevado de evacuações, sendo o mínimo de 3 vezes em um período de $24 \mathrm{~h}$ e em alguns casos pode haver ocorrência de muco e sangue. Elas são autolimitadas e duram até 14 dias. O problema desses quadros, é que sua evolução pode ocasionar desidratação de grau leve a grave. O não tratamento ou tratamento inadequado podem gerar além da desidratação, um distúrbio hidroeletrolítico podendo levar o paciente a óbito, principalmente quando há desnutrição associada. A diarreia aguda é considerada um problema de saúde pública em países em desenvolvimento e afeta pessoas de todas as faixas etárias, porém 
atinge principalmente crianças menores de 5 anos (Alfonso \& Bernal, 2019; Brasil, 2019).

Frequentemente, as diarreias têm origem em infecções gastrointestinais causadas por vírus, bactérias e protozoários. Porém, já são conhecidos alguns dos microrganismos que frequentemente são os responsáveis pela maioria dos episódios diarreicos. O rotavírus, por exemplo, é responsável por $40 \%$ de todas as admissões hospitalares por diarreia entre crianças menores de cinco anos em todo o mundo. Outros patógenos bacterianos muito conhecidos são E. coli, Shigella, Campylobacter e Salmonella com V. cholerae que estão mais associados a surtos epidêmicos (WHO, 2009).

Os países em desenvolvimento são os mais atingidos com esse problema pois, em sua maioria, tem precarização no fornecimento de água potável segura, saneamento básico, higiene e dispõem de um mau estado geral relacionado à saúde nutricional. Esses fatores se tornam uma complicação considerando que a maioria dos patógenos responsáveis pelas doenças diarreicas compartilham a mesma via de transmissão, sendo essa conhecida como transmissão fecal-oral, passando das fezes de uma pessoa até a boca de outra, a transmissão ocorre principalmente associada a ingestão de água e alimentos contaminados por esses patógenos (WHO, 2009).

A saúde da criança vem sendo alvo de políticas públicas e investimentos à medida que entende-se que é uma população mais suscetível a complicações por doenças devido a fragilidade própria do extremo de idade. Pensando nesse contexto, estratégias foram criadas a fim de promover menores taxas de internação hospitalar infantil. Desde 2015, o Ministério da Saúde investe no desenvolvimento de ações, estratégias e políticas voltadas para a primeira infância através da Política Nacional de Atenção Integral à Saúde da Criança (PNAISC). Essas ações são medidas preventivas importantes para a diminuição do número de internações hospitalares e mortalidade infantis (Oliveira et al., 2012; Brasil, 2015).

Esses investimentos em programas, iniciativas e políticas públicas que fortaleçam as ações em saúde e desenvolvimento saudável de crianças são importantes e trazem retorno para a sociedade, com menor gasto de internação futura, melhora na economia, além disso, contribui para reduzir as desigualdades sociais e é uma ferramenta de enfrentamento da pobreza, auxiliando na construção de uma sociedade com condições sociais e ambientais sustentáveis (Black et al., 2017).

Neste enfoque, a presente pesquisa apresenta como objeto de estudo o número de internações hospitalares por diarreias e gastroenterites de origem infecciosa e seus fatores associados em crianças de 0 a 4 anos em Macaé/RJ e tem como objetivos descrever e discutir o número de internações hospitalares por diarreias e gastroenterites de origem infecciosa em crianças de 0 a 4 anos em Macaé/RJ, comparado ao Estado do Rio de Janeiro e ao Brasil.

\section{Metodologia}

Este estudo foi desenvolvido na perspectiva da pesquisa epidemiológica e, portanto, possui abordagem quantitativa com análise retrospectiva a partir de fontes secundárias de dados disponíveis publicamente em sítios eletrônicos oficiais.

O estudo epidemiológico pesquisa a distribuição dos determinantes das doenças associadas à saúde de pessoas em determinada localização e tem como objetivo monitorar problemas de saúde para proposição de estratégias de controle para benefício da população estudada. A partir de informações específicas sobre um grupo populacional específico, em uma determinada localização geográfica e espaço temporal definidos, espera-se que os resultados possam retratar um olhar sobre a realidade que permita a utilização dos mesmos em grupos com características semelhantes (Gomes et al., 2005).

De acordo com Franco e Passos (2011), os estudos epidemiológicos implicam em transformações biológicas, antropológicas ou sociais, visto que é um método que estuda a identificação e análise do processo saúde-doença de forma coletiva. Franco e Passos afirma haver um certo consenso quanto as diferentes utilizações dos estudos epidemiológicos, e em geral, eles se propõem a: a) Identificar o problema de saúde na região através de levantamento histórico local; b) Projetar e avaliar possíveis impactos e relevância na comunidade; c) Identificar a população mais vulnerável e fatores de risco; d) Planejar ações e métodos em saúde a fim de intervir nos problemas identificados. As fontes de dados dos eventos estudados em 
epidemiologia, em sua maioria, são coletadas e armazenadas por órgãos relacionados a vigilância epidemiológica fornecidos publicamente para análise posterior.

Os dados de uma base secundária são aqueles que já foram anteriormente coletados, tabulados, ordenados e armazenados em bancos de dados. Este é um estudo com análise retrospectiva, onde examinam-se as amostras e dados coletados no passado (Fontelles et al., 2009). Os dados de interesse foram identificados, selecionados e sistematizados a partir dos dados brutos disponíveis no sítio da Internet do Departamento de Informática do Sistema Único de Saúde (DATASUS).

A coleta de dados ocorreu no período compreendido entre julho de 2020 e maio de 2021 e foi sistematizada a partir dos seguintes critérios: identificação dos dados populacionais, geográficos, etários e nosocomiais gerais; tratamento dos dados para seleção dos dados de interesse segundo os critérios de inclusão deste estudo, considerando o público-alvo em suas características (idade, local de residência, causas de internação hospitalar) e análise comparativa entre o município, o estado correspondente e a abrangência nacional.

Assim, foram construídos quadros, tabelas e gráficos para as informações referentes ao número de crianças de 0 a 4 anos que foram internadas por diarreia e gastroenterite de origem infecciosa no município de Macaé, no Estado do Rio de Janeiro e no Brasil no período de 2016 a 2020. Os dados selecionados foram organizados graficamente através da utilização do software Excel $365^{\circledR}$. A partir das informações sistematizadas foram estruturadas a discussão e análise dos mesmos.

\section{Resultados e Discussão}

Segundo os estudos de estimativa populacional a população abrangida pelo estudo, crianças de 0 a 4 anos no município de Macaé/RJ, apresentou um crescimento de 7,45\% entre os anos de 2016 e 2020, variando de 19.287 crianças em 2016 para 20.724 em 2020 (DATASUS, 2020). Assim, monitorar as causas de internação infantil nessa faixa etária no decorrer dos anos é importante pois elas são mais vulneráveis devido às fragilidades da idade relacionados à imunidade e a exposição a fatores de risco (Barbosa et al., 2017).

Os dados mostram que o município de Macaé, nos últimos 5 anos não apresentou regularidade nos números de internações, sendo 86 internações no ano de 2016, 82 em 2017, 123 em 2018, 206 em 2019 e 154 em 2020 . Todas estas, foram internações por algum tipo de doença infecciosa e parasitária. O Gráfico 1 aponta que a partir do ano de 2017, houve um crescimento expressivo no número de internações de crianças sendo 2019 o ano com maior número de casos nos últimos 5 anos, registrando queda em 2020. Destaca-se o fato do número de casos registrados no triênio (2017-2019) considerando um aumento de mais de $150 \%$ no registro das causas de internação. Outro aspecto, em destaque refere-se ao ano de 2020 que apesar de ter apresentado queda nos registros, estes ainda se mantiveram elevados se comparado ao período entre 2016 e 2017. 
Gráfico 1 - Internação por doenças infecciosas e parasitárias em crianças de 0 a 4 anos no município de Macaé/RJ (2016 2020).

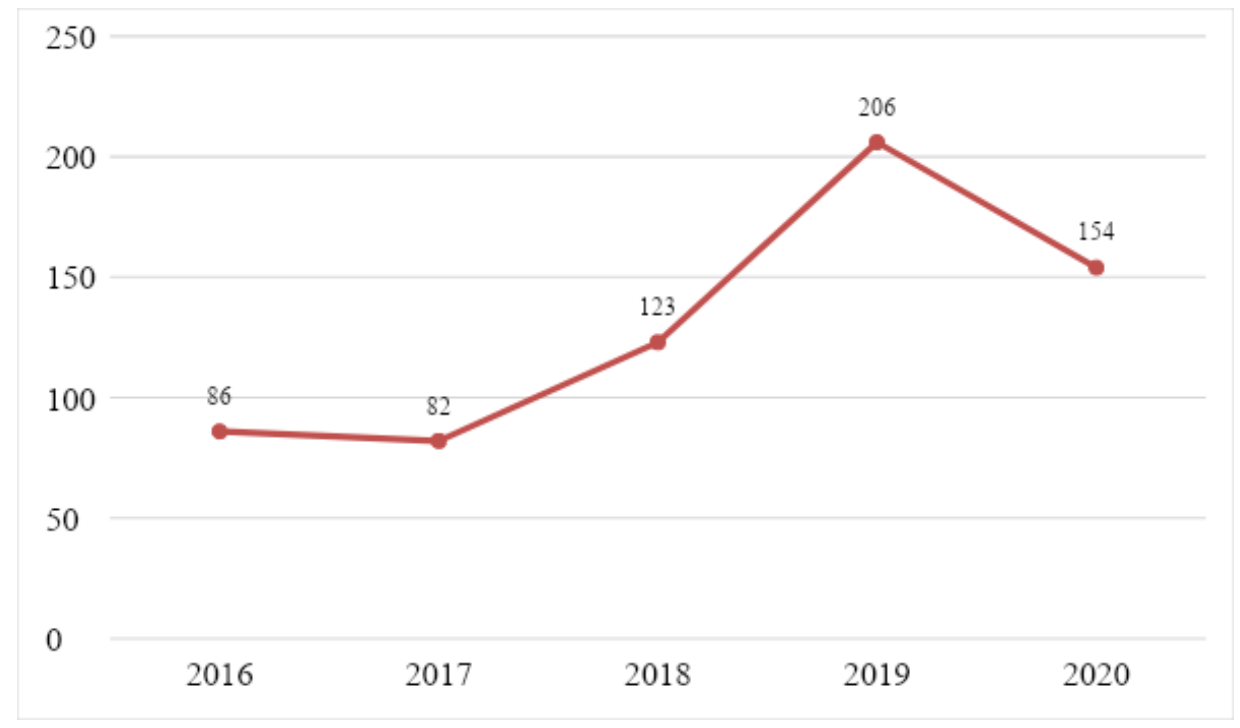

Fonte: Sistema de Internação Hospitalar do SUS (SIH-SUS). DATASUS. (2021).

Em 2020, ano no qual observou-se queda nos registros, foi marcado pela pandemia do novo coronavírus (COVID 19) que surgiu na cidade de Wuhan na China no final de 2019. E como trata-se de um vírus com um alto potencial de transmissão, medidas de distanciamento social, higiene e uso de máscaras foram ações fundamentais para a contenção e diminuição da circulação do patógeno. Devido ao isolamento social, muitas famílias passaram a ficar mais tempo dentro de suas casas e as crianças também foram afetadas por essas mudanças na rotina (Bittencourt, 2020).

Por passar mais tempo dentro em suas residências, as crianças durante o ano de 2020 ficaram menos expostas a condições de risco relacionadas ao convívio com outras crianças, compartilhamento de brinquedos e ambientes como escola, creches e locais de recreação infantil coletivos que poderiam torná-las mais suscetíveis ao adoecimento por doenças infecciosas intestinais.

Em contrapartida, entre as crianças que residem em ambientes sanitários inseguros, a exposição aumentou em decorrência do maior tempo que tiveram que passar nas suas residências, visto que no Brasil ainda há muitas habitações irregulares e sem acesso aos serviços de saneamento. Então, destaca-se o ambiente com fatores potenciais para o adoecimento de crianças por doenças diarreicas infecciosas (IBGE, 2020).

Além disso, a pandemia acentuou a desigualdade socioeconômica em todo o país, o que impactou diretamente no poder de compra e na alimentação da população. O último relatório disponibilizado pela Fundação Getúlio Vargas (FGV), em 2021, apontou que o número de cidadãos que vivem abaixo da linha da pobreza triplicou no último ano, atingindo aproximadamente 27 milhões de pessoas, o equivalente a 12,8\% da população (FGV, 2021).

$\mathrm{O}$ aumento da pobreza influenciou também a segurança alimentar dos brasileiros. Uma pesquisa conduzida pela Universidade Livre de Berlim em parceria com a Universidade Federal de Minas Gerais e a Universidade de Brasília revelou que 125,6 milhões de brasileiros passaram por situação de insegurança alimentar durante o último trimestre de 2020 . O acesso a alimentos importantes para a manutenção de uma dieta equilibrada também foi afetado visto que $44 \%$ da amostra da pesquisa reduziu o consumo de carne e $41 \%$ reduziram o consumo de frutas. No contexto da pandemia, foi constatada a redução de mais de $85 \%$ da ingesta de alimentos saudáveis entre os entrevistados em que a residência se encontrava em situação de insegurança alimentar (Galindo et al., 2021). 
Assim, é possível notar que esse cenário contribuiu com a suscetibilidade de adultos e crianças a comprometimentos em saúde visto que a manutenção de uma dieta balanceada é fundamental para assegurar um bom funcionamento das funções corporais. Esses fatores, quando associados à diminuição da procura por serviços de saúde em função do medo da população em contrair a doença, podem proporcionar um cenário que influencia o número de internações infantis.

Então, dentre as causas de internações, é importante monitorar as que são derivadas de patógenos que são transmitidos através de água e alimentos contaminados, pois isso é um indicador importante para um bom diagnóstico situacional e planejamento em saúde. Através dos dados obtidos pelo Sistema de Internação Hospitalar do Sistema Único de Saúde (SIHSUS) no DATASUS, foram identificados números reduzidos de internações infantis por doenças diarreicas e gastroenterite de origem infecciosa no município de Macaé/RJ, no período estudado, como descrito a seguir.

Tabela 1 - Internação de crianças de 0 a 4 anos por diarreia e gastroenterite de origem infecciosa no município de Macaé/RJ $(2016-2020)$.

\begin{tabular}{|c|c|c|c|c|c|c|c|c|c|c|}
\hline \multirow{2}{*}{$\begin{array}{c}\text { Causas de } \\
\text { Internação }\end{array}$} & \multicolumn{2}{|c|}{2016} & \multicolumn{2}{|c|}{2017} & \multicolumn{2}{|c|}{2018} & \multicolumn{2}{|c|}{2019} & \multicolumn{2}{|c|}{2020} \\
\hline & $\mathrm{N}$ & $\%$ & $\mathrm{~N}$ & $\%$ & $\mathrm{~N}$ & $\%$ & $\mathrm{~N}$ & $\%$ & $\mathrm{~N}$ & $\%$ \\
\hline $\begin{array}{c}\text { Outras doença } \\
\text { infecciosas } \\
\text { intestinais }^{1}\end{array}$ & 4 & 4.65 & 4 & 4.87 & 3 & 2.43 & 3 & 1.45 & 1 & 0.64 \\
\hline $\begin{array}{c}\text { Outras } \\
\text { helmintíases }\end{array}$ & - & - & 2 & 2.43 & 1 & 0.81 & - & - & 1 & 0.64 \\
\hline $\begin{array}{c}\text { Doenças } \\
\text { infecciosas }^{2}\end{array}$ & 82 & 95.34 & 75 & 92.68 & 119 & 96.74 & 203 & 98.54 & 152 & 98.7 \\
\hline $\begin{array}{c}\text { Total de } \\
\text { doenças } \\
\text { infecciosas e } \\
\text { parasitárias }\end{array}$ & 86 & 100 & 82 & 100 & 123 & 100 & 206 & 100 & 154 & 100 \\
\hline
\end{tabular}

${ }^{1}$ Essa categoria inclui todas as infecções por Escherichia coli, enterite por Campylobacter, Yersinia enterocolítica, Clostridium difficile e infecções bacterianas não especificadas excluindo a diarreia e gastroenterite de origem infecciosa presumível (DATASUS, 2008).

2 Essa categoria se refere a doenças infecciosas se referem à todas as outras patologias de origem infecciosa e parasitárias que não causam diarreia e gastroenterite. Fonte: Sistema de Internação Hospitalar do SUS (SIH/SUS). DATASUS. (2021).

Das internações por doenças infecciosas e parasitárias no município de Macaé, só houve registro de outras helmintíases e outras doenças infecciosas intestinais. Apenas 4,65\% dos casos em 2016 eram por outras doenças infecciosas intestinais. No ano de 2017, as internações por outras doenças infecciosas intestinais foram 4,87\% sendo 2,43\% por outras helmintíases; em 2018 diminuiu para 2,43\% por outras doenças infecciosas intestinais e 0,81\% para outras helmintíases. Em 2019 apenas $1,45 \%$ das internações estudadas foram por outras doenças infecciosas intestinais e no ano de 2020 apenas $0,64 \%$ foram por outras doenças infecciosas intestinais e $0,64 \%$ para outras helmintíases. As doenças infecciosas não relacionadas a gastroenterite e diarreia apresentam as maiores taxas percentuais variando entre 75 e $98.7 \%$ ao longo dos anos.

Considerando o ano de 2020, das 20.724 crianças de 0 a 4 anos que residiam no município, $0,74 \%$ delas foram internadas por algum tipo de doença infecciosa ou parasitária e apenas $0,009 \%$ estavam relacionadas à doença diarreica. Não houve registo do número de internações para Shigelose, Amebíase, Cólera e Esquistossomose no município e isso dificultou a análise dos casos reais que ocorreram na cidade visto que os dados estaduais e nacionais apontaram um cenário diferente do que o município apresenta. 
Os dados epidemiológicos do município de Macaé sinalizaram padrões distintos quando comparados aos dados estaduais e nacionais, apesar de apresentar características sanitárias urbanas com impactos na saúde humana. Este fato pode sugerir diversas questões que necessitam de investigações no que se refere a dados de internação infantil por doenças diarreicas e gastroenterite de origem infecciosa relacionados com a ingestão de água e alimentos contaminados.

$\mathrm{Na}$ avaliação das características epidemiológicas nacionais foi possível identificar que o Brasil é um país que apresenta disparidades socioeconômicas e culturais entre as regiões, essa desigualdade também repercute no acesso aos serviços de saúde e de saneamento básico. As regiões do país onde há maior concentração da população negra são as áreas onde há maior dificuldade no acesso aos serviços de saúde e isso reverbera na saúde da criança. A região sudeste é a mais desenvolvida do país por abrigar dois estados que estão entre as maiores economias do país: São Paulo e Rio de Janeiro. Por isso, de acordo com os últimos relatórios da Secretaria Nacional de Saneamento (SNS) com o Ministério de Desenvolvimento Regional de 2019, ela também é a região que recebe mais serviços de saneamento com cobertura de $91,1 \%$ para acesso a água tratada, 79,5\% para tratamento de esgoto e 96,2\% para manejo de resíduos sólidos urbanos apesar da heterogeneidade de disponibilidade de serviços (Dantas et al., 2021; Brasil, 2020a; Brasil, 2020b; IBGE, 2018).

Comparar os dados municipais com o cenário estadual e nacional é importante para a monitorização de situações urgentes e investigação de possíveis problemas locais que influenciam na saúde das crianças. Os dados do estado do Rio de Janeiro não seguiram um padrão, porém, desde o ano de 2017 ocorreu um aumento gradativo no número de internações infantis nos anos subsequentes que pode ser explicado pelo real aumento das internações, indicando que mais crianças ficaram expostas a ambientes e fatores de risco ou pela melhora na qualificação dos registros ocorridos neste período.

Os números referentes a doenças infecciosas se referem à todas as outras patologias de origem infecciosa e parasitárias que não causam diarreia e gastroenterite, por isso, elas representam a maioria do número de casos em todos os cenários, por abrigar a maior parte das doenças registradas como a tabela a seguir evidencia.

Tabela 2 - Dados sobre internação de crianças de 0 a 4 anos por diarreia e gastroenterite de origem infecciosa no estado do Rio de Janeiro (2016 - 2020).

2016

\begin{tabular}{|c|c|c|c|c|c|c|c|c|c|c|}
\hline Causas de Internação & $\mathrm{N}$ & $\%$ & $\mathrm{~N}$ & $\%$ & $\mathrm{~N}$ & $\%$ & $\mathrm{~N}$ & $\%$ & $\mathrm{~N}$ & $\%$ \\
\hline Shiguelose & 2 & 0.02 & 1 & 0.01 & 1 & 0.01 & 2 & 0.02 & 1 & 0.08 \\
\hline Amebíase & 2 & 0.02 & 1 & 0.01 & 3 & 0.03 & - & - & 1 & 0.08 \\
\hline Cólera & 3 & 0.03 & 1 & 0.01 & 3 & 0.03 & 2 & 0.02 & 1 & 0.08 \\
\hline $\begin{array}{c}\text { Outras doenças } \\
\text { infecciosas intestinais }\end{array}$ & 993 & 9.26 & 910 & 9.08 & 1141 & 11.15 & 1317 & 11.54 & 1410 & 11.09 \\
\hline Esquistossomose & 1 & 0.01 & - & - & - & - & 2 & 0.02 & - & - \\
\hline Outras helmintíases & 45 & 0.42 & 46 & 0.46 & 28 & 0.27 & 39 & 0.34 & 14 & 0.1 \\
\hline Doenças infecciosas & 9671 & 90.23 & 9059 & 90.42 & 9052 & 88.5 & 10054 & 88.06 & 11318 & 88.36 \\
\hline $\begin{array}{l}\text { Total de doenças } \\
\text { infecciosas e } \\
\text { parasitárias }\end{array}$ & 10717 & 100 & 10018 & 100 & 10228 & 100 & 11416 & 100 & 12808 & 100 \\
\hline
\end{tabular}


De acordo com a Tabela 2, 2016 apresentou ao todo 1.046 internações infantis por alguma doença diarreica de origem infecciosa e parasitária (shiguelose, amebíase, cólera, outras doenças infecciosas intestinais, esquistossomose e outras helmintíases), tendo uma diminuição em 2017 com 959 internações sofrendo um aumento de 2018 até o ano de 2020 apresentando 1.176 internações em 2018, 1.362 internações em 2019 e 1427 internações em 2020. Assim, este grupo de patologias representou um acometimento de mais de $10 \%$ das internações infantis específicas. Apesar de ser menor em relação a doenças infecciosas, afetou um número grande de crianças, gerando consequências diversas para a criança e sua família.

Os dados mostram que mesmo o Sudeste sendo a região com maior acesso aos serviços de saneamento ainda sofreu com o adoecimento de crianças por doenças sensíveis às condições ambientais e sanitárias, porém, nos últimos anos foi visto poucas internações por amebíase, shigelose, cólera e esquistossomose. Esses números de internações podem estar relacionados com a melhora nas condições de higiene e intervenção precoce que evitou a internação dessas crianças, além da melhora na cobertura dos serviços de saneamento (McQuade et al., 2020; Souza et al., 2019).

O Brasil não acompanhou a tendência do Rio de Janeiro e de Macaé no que tange ao número total de casos pois 2016 foi o ano que teve maior registo no número de internações infantis, já nos anos de 2017 até 2019, ocorreu um aumento no número de casos indo de 163.958 internações em 2017 para 167.796 em 2019. O ano de 2020 teve uma diminuição considerável em relação aos anos anteriores com redução de $25,7 \%$ em relação ao ano anterior.

Tabela 3 - Dados sobre internação de crianças de 0 a 4 anos por diarreia e gastroenterite de origem infecciosa no Brasil (2016 $-2020)$.

\begin{tabular}{|c|c|c|c|c|c|c|c|c|c|c|}
\hline \multirow[b]{2}{*}{$\begin{array}{l}\text { Causas de } \\
\text { Internação }\end{array}$} & \multicolumn{2}{|c|}{2016} & \multicolumn{2}{|c|}{2017} & \multicolumn{2}{|c|}{2018} & \multicolumn{2}{|c|}{2019} & \multicolumn{2}{|c|}{2020} \\
\hline & $\mathrm{N}$ & $\%$ & $\mathrm{~N}$ & $\%$ & $\mathrm{~N}$ & $\%$ & $\mathrm{~N}$ & $\%$ & $\mathrm{~N}$ & $\%$ \\
\hline Shiguelose & 51 & 0.03 & 30 & 0.09 & 40 & 0.02 & 49 & 0.03 & 26 & 0.02 \\
\hline Amebíase & 692 & 0.39 & 381 & 0.23 & 292 & 0.17 & 343 & 0.2 & 156 & 0.12 \\
\hline Cólera & 189 & 0.1 & 191 & 0.17 & 156 & 0.09 & 166 & 0.09 & 120 & 0.09 \\
\hline $\begin{array}{c}\text { Outras doenças } \\
\text { infecciosas intestinais }\end{array}$ & 43976 & 23.42 & 34008 & 20.74 & 32679 & 19.62 & 30897 & 18.41 & 17724 & 14.21 \\
\hline Esquistossomose & 5 & 0.003 & 8 & 0.005 & 12 & 0.007 & 10 & 0.005 & 10 & 0.01 \\
\hline Outras helmintíases & 326 & 0.17 & 322 & 0.19 & 320 & 0.19 & 312 & 0.18 & 198 & 0.16 \\
\hline Doenças infecciosas & 142525 & 75.9 & 129018 & 78.68 & 133068 & 79.88 & 136019 & 81.06 & 106691 & 85.54 \\
\hline $\begin{array}{l}\text { Total de doenças } \\
\text { infecciosas e } \\
\text { parasitárias }\end{array}$ & 187764 & 100 & 163958 & 100 & 166567 & 100 & 167796 & 100 & 124725 & 100 \\
\hline
\end{tabular}

Fonte: Sistema de Internação Hospitalar do SUS (SIH/SUS). DATASUS. (2021).

Desde 2016, os números de internações por doenças diarreicas de origem infecciosa e parasitária (shiguelose, amebíase, cólera, outras doenças infecciosas intestinais, esquistossomose e outras helmintíases), no país diminuíram visto que houve 45.239 internações em 2016, 34.940 internações em 2017, 33.499 internações em 2018, 31.777 internações em 2019 e 
18.234 internações em 2020. Isso pode estar relacionado a políticas públicas e estratégias adotadas em âmbito nacional que tem trazido resolutividade para o problema de adoecimento de crianças por causas relacionadas à alimentação e condições sanitárias de risco, porém, mesmo com os avanços no fornecimento de serviços de saneamento básico e maior acesso a esses serviços, muitas crianças sofreram por doenças evitáveis.

No município de Macaé, foi visto um panorama diferente do cenário apresentado no estado do Rio de Janeiro e no Brasil pois houve poucos casos na cidade referentes a internação por doenças diarreicas. Contudo, é necessário ponderar que a rede pública de saúde no Brasil se organiza de forma a evitar o serviço hospitalar, e as doenças infecciosas intestinais são condições sensíveis à Atenção Primária à Saúde (APS), porém, segundo dados fornecidos pela Secretaria de Atenção Primária à Saúde (SAPS), a cobertura estimada da Atenção Básica pelo número de habitantes em Macaé no mês de dezembro de 2020 era apenas de 48,10\%, ou seja, ainda havia uma grande parcela da população descoberta pelo serviço de saúde que é considerado porta de entrada para casos de diarreia e gastroenterite em crianças (SAPS, 2021; Brasil, 2008). Desta forma, tornou-se de difícil compreensão a redução do número de internações em uma localidade com uma cobertura em assistência primária reduzida.

A APS, em geral, é um conjunto de ações e medidas aplicadas em âmbito individual e coletivo a fim de promover saúde e prevenir agravos fornecendo um cuidado integral aos usuários. Há muitas estratégias governamentais para implementação da APS, uma delas é a Estratégia de Saúde da Família (ESF), que oferece diversos serviços às comunidades através das Unidades de Saúde da Família (USF). As ESF têm papel fundamental no diagnóstico precoce, na formulação de intervenções e na avaliação em saúde no que tange às doenças diarreicas, além de minimizar os riscos relacionados à deficiência da prestação de serviços sanitários nas comunidades. A literatura aponta que ampliar esses serviços têm impactos positivos na saúde da criança, pois as ações das unidades são planejadas de acordo com as especificidades regionais, o que gera maior resolutividade aos problemas locais que influenciam na saúde da criança (Brasil, 2012; Macedo et al., 2019; Aguiar et al., 2020).

Devido à baixa cobertura da atenção básica no município, infere-se que houve uma invisibilização dos casos reais de crianças acometidas por infecções intestinais, porém, outro fator que deve ser analisado é que possa haver falhas nos diagnósticos finais relacionando-os a causas como a desnutrição que é uma complicação comum em casos de diarreia, principalmente quando há demora no tratamento ou tratamento inadequado. Desta forma, monitorar os casos de internação por complicações da diarreia também é importante para avaliar a localidade como descrito na tabela abaixo.

Tabela 4 - Dados sobre internação de crianças de 0 a 4 anos por desnutrição no município de Macaé/RJ (2016- 2020).

\begin{tabular}{|c|c|c|c|c|c|c|c|c|c|c|}
\hline \multirow[b]{2}{*}{ Causas de Internação } & \multicolumn{2}{|c|}{2016} & \multicolumn{2}{|c|}{2017} & \multicolumn{2}{|c|}{2018} & \multicolumn{2}{|c|}{2019} & \multicolumn{2}{|c|}{2020} \\
\hline & $\mathrm{N}$ & $\%$ & $\mathrm{~N}$ & $\%$ & $\mathrm{~N}$ & $\%$ & $\mathrm{~N}$ & $\%$ & $\mathrm{~N}$ & $\%$ \\
\hline Desnutrição & 2 & 50 & 1 & 25 & 4 & 80 & 15 & 68.18 & 23 & 92 \\
\hline $\begin{array}{c}\text { Outras doenças metabólicas e } \\
\text { endócrinas }\end{array}$ & 2 & 50 & 3 & 75 & 1 & 20 & 7 & 31.8 & 2 & 8 \\
\hline $\begin{array}{c}\text { Total de doenças metabólicas e } \\
\text { endócrinas }\end{array}$ & 4 & 100 & 4 & 100 & 5 & 100 & 22 & 100 & 25 & 100 \\
\hline
\end{tabular}


Em 2016, houve 2 internações por desnutrição, 1 internação em 2017, e a partir desse ano, houve um aumento expressivo nas internações por desnutrição nos anos subsequentes sendo 4 internações em 2018, 15 internações em 2019 e 23 internações em 2020. Neste último ano, as internações por desnutrição corresponderam a 92,0\% do total de doenças. Mesmo que não seja possível apontar diretamente a relação da hospitalização por desnutrição no município com as doenças intestinais, faz-se necessário o acompanhamento e o cruzamento de dados mais específicos para que eles sejam explorados e depurados de forma adequada.

A inserção de um serviço de referência para atendimento especializado em saúde da criança no município em 2016 também pode ter contribuído para a prevenção de agravos por doenças diarreicas, diagnóstico e tratamento precoces e na identificação e encaminhamento de casos resolvíveis na atenção primária em saúde que se refletem no número de casos hospitalares. Além disso, para este estudo foram analisados apenas os casos que receberam atendimento em saúde em unidades do SUS, portanto, foram excluídos os atendimentos em instituições privadas.

As práticas culturais domiciliares no manejo das doenças diarreicas agudas também são fatores importantes para a compreensão sobre as baixas taxas de internação municipal. A administração de soros caseiros, chás, bebidas ritualísticas são práticas amplamente adotadas em território nacional que podem influenciar nos casos graves que precisam de atendimento hospitalar.

Além disso, a incorporação da vacinação contra o rotavírus no Programa Nacional de Imunização (PNI) e no calendário vacinal em Macaé em 2006 também contribuiu para a diminuição dos casos de diarreia no município visto que as rotaviroses são conhecidas por serem uma das grandes responsáveis por doenças gastroentéricas graves na infância, por isso é de suma importância a adesão ao calendário vacinal adequadamente (Brasil, 2014).

Mesmo com todos esses pontos favoráveis à redução das internações infantis no município, as condições observadas no município sugerem outro cenário relacionado à saúde da criança, por isso, é necessário investigar também os fatores associados aos quadros de internação, sendo essas, as condições sanitárias locais e a segurança alimentar nos domicílios onde as crianças residem.

Assim, o monitoramento periódico das internações infantis por doenças gastrointestinais de origem infecciosa é de suma importância para o planejamento em saúde do município visto que esses dados, se forem depurados de adequadamente, sinalizam problemas emergentes que influenciam na qualidade de vida e na saúde de crianças e seus cuidadores. Além disso, se faz necessário para a avaliação e adequação de medidas tomadas e auxílio no preparo de novas medidas preventivas. Por isso, é importante garantir que os registros hospitalares sejam feitos de forma correta, assim como o diagnóstico e o tratamento adequados.

\section{Conclusão}

O município estudado apresentou baixas taxas de internações hospitalares infantis quando comparados aos dados estaduais e nacionais. Além disto, as informações sobre as internações quando fornecidas de forma isolada, sugerem situações que podem ser explicadas quando associadas a outros dados que estejam relacionados com os números estudados, por isso, analisar os dados fornecidos publicamente sobre saneamento básico, alimentação infantil, dados socioeconômicos e atendimentos em saúde são fundamentais para melhor entendimento do cenário municipal no que se refere ao adoecimento de crianças por DDA.

A vigilância em saúde é uma ferramenta utilizada para identificar e analisar as condições de risco de um local e com base nos dados, articular ações a fim de intervir nos condicionantes transversais ao processo saúde-doença e propor novas estratégias para melhorar as condições ambientais e atuar na prevenção de doenças, principalmente as que atingem a população infantil. 
Nesse contexto, os estudos e análises epidemiológicas podem ser feitos a partir de dados disponibilizados publicamente e são uma das formas de executar ações de vigilância em saúde. Monitorar e avaliar a frequência, distribuição e a causa dos problemas em saúde é fundamental para prestar um cuidado integral, por isso, a inclusão de dados precisos nos sistemas de informações é fundamental para melhor apuração dos problemas locais e para o planejamento adequado de ações que visem a melhoria e ajuste de políticas públicas que influenciam na qualidade de vida da população.

Devido a importância dos dados nos sistemas de informações e para que esse processo seja feito de forma segura, é necessário promover condições ideais de trabalho para os profissionais que prestam assistência, visto que esses dados brutos derivam, em sua maioria, da vigilância e monitoramento de rotina. Por isso, investir em qualificação profissional sobre os sistemas de informações, instruir sobre como inserir dados precisos nas plataformas, promover tempo para inserção das informações, recursos adequados para trabalho e estruturas adequadas para o funcionamento dos sistemas que usam rede de dados são fundamentais para diminuir imprecisões nos resultados epidemiológicos.

A DDA é um problema de saúde pública multifatorial que precisa ser resolvida com planejamentos de cuidados efetivos a partir de ações multiprofissionais. Por isso, a vigilância em saúde e análise dos dados brutos precisa ser avaliada por diversos serviços e especialidades que contribuem para uma ação holística e integral na atenção à saúde da criança. Nesse contexto, o enfermeiro tem autonomia e competência para atuar na avaliação, identificação, monitorização de grau de exposição a fatores danosos à saúde e auxiliar na idealização e execução de planos estratégicos para controle de fatores de risco à saúde infantil.

A ausência quantitativa de dados sobre internações infantis por gastroenterites e diarreia não demonstra a ausência das ocorrências da patologia e suas consequências no cenário local, sobretudo quando analisados os fatores associados e o cenário estadual e nacional descritos. Ao contrário, evidencia que estas informações podem estar invisibilizadas por ausência de qualificação da informação registrada ou do próprio registro nas bases de dados. Portanto, a atenção a esta realidade torna-se um importante enfoque a ser avaliado para a atenção à saúde infantil local.

Assim, considera-se importante investir em novos estudos ampliando a análise para inclusão de dados sobre internação infantil nos serviços privados a serem acrescidos na avaliação da magnitude dos casos de internação no município. São necessárias avaliações que ampliem a discussão incluindo o SUS e a as unidades que integram a rede de saúde suplementar local. Os fatores relacionados ao adoecimento de crianças por doenças diarreicas e gastroenterite de origem infecciosa, como a segurança alimentar, saneamento básico, meio ambiente e dados socioeconômicos devem ser considerados na atenção à saúde infantil de modo a subsidiar o enfermeiro e demais membros da equipe de saúde no cuidado, ampliando os conhecimentos científicos referentes a temática e contribuindo para melhora nos indicadores de morbidade e mortalidade na infância, por doenças evitáveis.

\section{Referências}

Aguiar, K. C. G. D., Cohen, S. C., Maciel, E. M. G. D. S., \& Kligerman, D. C. (2020). Fatores de risco para ocorrência de diarreia em crianças residentes na Ilha de Guaratiba (RJ). Saúde em Debate, 44, 205-220. https://doi.org/10.1590/0103-1104202012415

Alfonso, E. P. \& Bernal, D. H. (2019). La Enfermedad Diarreica Aguda. Revista Cubana de Pediatria, 91 (4). http://www.revpediatria.sld.cu/index.php/ped/article/view/928/467

Barbosa, S. F. A., Costa, F. M. D., \& Vieira, M. A. (2017). Causas de hospitalização de crianças: uma revisão integrativa da realidade brasileira. Espaço para Saúde, 18(2), 129-137. https://espacoparasaude.fpp.edu.br/index.php/espacosaude/article/view/245

Bittencourt, R. N. (2020). Pandemia, isolamento social e colapso global. Revista Espaço Acadêmico, 19(221), 168-178. https://periodicos.uem.br/ojs/index.php/EspacoAcademico/article/view/52827

Black, M. M., Walker, S. P., Fernald, L., Andersen, C. T., DiGirolamo, A. M., Lu, C., McCoy, D. C., Fink, G., Shawar, Y. R., Shiffman, J., Devercelli, A. E., Wodon, Q. T., Vargas-Barón, E., Grantham-McGregor, S., \& Lancet Early Childhood Development Series Steering Committee. (2017). Early childhood development coming of age: science through the life course. Lancet, 389(10064), 77-90. https://doi.org/10.1016/S0140-6736(16)31389-7 
Brasil. (2008). Ministério da Saúde. Secretaria de Atenção à Saúde. Portaria $n^{\circ}$ 221, de 17 de abril de 2008. Sistema de Legislação da Saúde. https://bvsms.saude.gov.br/bvs/saudelegis/sas/2008/prt0221_17_04_2008.html

Brasil. (2012). Ministério da Saúde. Secretaria de Atenção à Saúde. Departamento de Atenção Básica. Política Nacional de Atenção Básica. http://189.28.128.100/dab/docs/publicacoes/geral/pnab.pdf

Brasil. (2014). Ministério da Saúde. Secretaria de Vigilância em Saúde e Departamento de Vigilância das Doenças Transmissíveis. Manual de vigilância epidemiológica de eventos adversos pós-vacinação. https://bvsms.saude.gov.br/bvs/publicacoes/manual_vigilancia_epidemiologica_eventos_adversos_pos_vacinacao.pdf

Brasil. (2015). Ministério da Saúde. Gabinete do Ministro. Portaria $n^{o}$ 1.130, de 5 de agosto de 2015 . http://bvsms.saude.gov.br/bvs/saudelegis/gm/2015/prt1130_05_08_2015.html

Brasil. (2019). Ministério da Saúde. Secretaria de Vigilância em Saúde e Coordenação-Geral de Desenvolvimento da Epidemiologia em Serviços. Guia de vigilância em saúde: volume único. (3 ${ }^{\mathrm{a}}$. Ed.). https://bvsms.saude.gov.br/bvs/publicacoes/guia_vigilancia_saude_3ed.pdf

Brasil. (2020a). Ministério do Desenvolvimento Regional. Secretaria Nacional de Saneamento (SNS). Sistema Nacional de Informações sobre Saneamento. $25^{\circ}$ Diagnóstico dos $\quad$ Serviços $\quad$ de $\quad$ Água $\quad$ Esgotos. http://snis.gov.br/downloads/diagnosticos/ae/2019/Diagn\%C3\%B3stico_SNIS_AE_2019_Republicacao_31032021.pdf

Brasil. (2020b). Ministério do Desenvolvimento Regional. Secretaria Nacional de Saneamento (SNS). Sistema Nacional de Informações sobre Saneamento. $18^{\circ}$ Diagnóstico do Manejo de Resíduos Sólidos Urbanos. http://snis.gov.br/downloads/diagnosticos/rs/2019/Diagnostico_RS2019.pdf

Dantas, M. N. P., Souza, D. L. B. D., Souza, A. M. G. D., Aiquoc, K. M., Souza, T. A. D., \& Barbosa, I. R. (2021). Fatores associados ao acesso precário aos serviços de saúde no Brasil. Revista Brasileira de Epidemiologia, 24. https://doi.org/10.1590/1980-549720210004

Departamento de Informática do Sistema Único de Saúde (DATASUS). (2008). CID - 10: A00-A09 Doenças infecciosas intestinais. http://www2.datasus.gov.br/cid10/V2008/WebHelp/a00_a09.htm

Departamento de Informática do Sistema Único de Saúde (DATASUS). (2020). Tecnologia da Informação a Serviço do SUS. https://datasus.saude.gov.br/informacoes-de-saude-tabnet/

Departamento de Informática do Sistema Único de Saúde (DATASUS). (2021). Tecnologia da Informação a Serviço do SUS. https://datasus.saude.gov.br/informacoes-de-saude-tabnet/

Franco, L. J., Passos, A. D. C. (2011). Fundamentos de Epidemiologia. Editora Manole Ltda, Barueri, SP - 2ª edição.

Fontelles, M. J., Simões, M. G., Farias, S. H., \& Fontelles, R. G. S. (2009). Metodologia da pesquisa científica: diretrizes para a elaboração de um protocolo de pesquisa. Revista Paraense de Medicina, 23(3), 1-8.

Fundação Getúlio Vargas (FGV). (2020). Efeitos da pandemia sobre o mercado de trabalho brasileiro: Desigualdades, ingredientes trabalhistas e o papel da jornada. Centro de Políticas Sociais da FGV. https://cps.fgv.br/pesquisas/efeitos-da-pandemia-sobre-o-mercado-de-trabalho-brasileiro

Galindo, E., Teixeira, M. A., de Araújo, M., Motta, R., Pessoa, M., Mendes, L., \& Rennó, L. (2021). Efeitos da pandemia na alimentação e na situação da

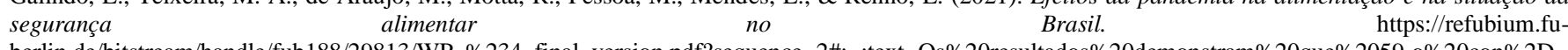
berlin.de/bitstream/handle/fub188/29813/WP_\%234_final_version.pdf?sequence=2\#: :text=Os\%20resultados\%20demonstram\%20que\%2059,o\%20con\%2D $\% 20$ sumo $\% 20 \mathrm{de} \% 20$ frutas.

Gomes, M. D. M., Li, L. M., \& Carvalho, V. N. (2005). Estudos epidemiológicos. Journal of Epilepsy and Clinical Neurophysiology, 11, 16-19. https://doi.org/10.1590/S1676-26492005000500004

Instituto Brasileiro de Geografia e Estatística (IBGE). (2018). Produto Interno Bruto dos Municípios. https://www.ibge.gov.br/estatisticas/economicas/contasnacionais/9088-produto-interno-bruto-dos-municipios.html?=\&t=resultados

Instituto Brasileiro de Geografia e Estatística (IBGE). (2020). Síntese de indicadores sociais: uma análise das condições de vida da população brasileira 2020. https://biblioteca.ibge.gov.br/visualizacao/livros/liv101760.pdf

Macedo, J. C. B., Arcêncio, R. A., Wolkers, P. C. B., Ramos, A. C. V., Toninato, A. P. C., \& Furtado, M. C. D. C. (2019). Fatores associados a pneumonias e diarreia em crianças e qualidade da atenção primária à saúde. Texto \& Contexto Enfermagem, 28, e20180225. http://dx.doi.org/10.1590/1980-265X-TCE2018-0225

McQuade, E. T. R., Shaheen, F., Kabir, F., Rizvi, A., Platts-Mills, J. A., Aziz, F., Kalam, A., Qureshi, S., Elwood, S., Liu, J., Lima, A., Kang, G., Bessong, P., Samie, A., Haque, R., Mduma, E. R., Kosek, M. N., Shrestha, S., Leite, J. P., Bodhidatta, L., ... \& Iqbal, N. T. (2020). Epidemiology of Shigella infections and diarrhea in the first two years of life using culture independent diagnostics in 8 low-resource settings. PLOS Neglected Tropical Diseases, 14(8), e0008536. https://doi.org/10.1371/journal.pntd.0008536

Oliveira, B. R. G., Vieira, C. S., Furtado, M. C. C., Mello, D. F. \& Lima, R. A. G. (2012). Perfil de morbidade de crianças hospitalizadas em um hospital público: implicações para a Enfermagem. Revista Brasileira de Enfermagem, 65 (4), 586-593. https://doi.org/10.1590/S0034-71672012000400006

Secretaria de Atenção Primária em Saúde (SAPS). (2021, maio). e-Gestor Atenção Básica. Informação e Gestão da Atenção Básica. Cobertura da Atenção Básica. https://egestorab.saude.gov.br/paginas/acessoPublico/relatorios/relHistoricoCoberturaAB.xhtml

Souza, C. S., Bandeira, L. L. B., Mariano, A. K. N. O., Nunes, M. D. P. S. F., \& de Souza Neto, J. D. (2019). Amebíase no contexto da emergência: análise do perfil de internações e morbimortalidade nos Estados brasileiros em 5 anos. Revista da Sociedade Brasileira de Clínica Médica, 17(2), 66-70. https://www.sbcm.org.br/ojs3/index.php/rsbcm/article/view/609 
Research, Society and Development, v. 11, n. 1, e39211125024, 2022

(CC BY 4.0) | ISSN 2525-3409 | DOI: http://dx.doi.org/10.33448/rsd-v11i1.25024

The United Nations Inter-agency Group for Child Mortality Estimation (UN IGME). (2019). Levels \& Trends in Child Mortality Estimates developed by the UN Inter-agency Group for Child Mortality Estimation. Fundo de Emergência das Nações Unidas para a Infância (UNICEF): Nova York. https://www.unicef.org/reports/levels-and-trends-child-mortality-report-2019

World Health Organization (WHO). (2009). Diarrhoea: Why children are still dying and what can be done. United Nations Children's Fund. World Health Organization.https://apps.who.int/iris/bitstream/handle/10665/44174/9789241598415_eng.pdf;jsessionid=10C0C84BAEC0D22B0F8E86F039276FEA?seque nce $=1$ 